\title{
Retinal vessel model fabricated on a curved surface structure for a simulation of microcannulation
}

\author{
Takeshi Hayakawa $^{1 *+} \mathbb{0}$, Ippei Kato ${ }^{1 \dagger}$, Fumihito Arai $^{1 \dagger}$, Mamoru Mitsuishi ${ }^{2}$, Naohiko Sugita ${ }^{2}$, Kanako Harada ${ }^{2}$, \\ Shinichi Tanaka², Yasuo Noda ${ }^{3}$ and Takashi Ueta ${ }^{3}$
}

\begin{abstract}
A remarkable number of vitreoretinal surgeries are performed each year despite their difficulty. As a result, a high demand exists for a mock-up simulator of retinal vessels to simulate these surgeries. Thus, we propose an artificial retinal vessel model for simulating microcannulation surgery. Using laser lithography, polydimethylsiloxane molding, and hydraulic transfer techniques, we fabricated microchannels approximately $\simeq 10 \mu \mathrm{m}$ on a 24-mm-diameter curved surface structure that mimics the human eye. In the fabrication, the channel size and wall thickness were controlled to mimic a touch of retinal vessels, which gives important information for microcannulation. We succeeded in fabrication of the proposed model and liquid circulates within the microchannels of this model without leaking. Furthermore, we demonstrated a simulation of microcannulation and measurement of applied force to the model during the simulation using a force sensor placed at the bottom of the model. The results of such experiments are useful to quantitatively evaluate medical techniques.
\end{abstract}

Keywords: Surgical simulator, Retinal vessel, 3D microfabrication

\section{Background}

Vitreoretinal surgeries have become quite common over the last few decades. For example, approximately 300,000 operations are conducted annually in Japan, and this number is predicted to increase because of the aging population. One such operation is called microcannulation, which is proposed to patients with central retinal vein occlusion [1]. In microcannulation, a surgeon inserts a micropipette into the eyeball and reaches the eye fundus, which is a curved concave structure (Fig. 1a). Next, the surgeon finds the occluded vein on the fundus and, via the micropipette, injects a thrombolytic drug into the vein to dissolve the clots. This operation requires mature surgical skills because the retinal vessel is less than $100 \mu \mathrm{m}$ in diameter (Fig.1b), This technique is used

\footnotetext{
*Correspondence: t-hayakawa@mech.nagoya-u.ac.jp

†Takeshi Hayakawa, Ippei Kato and Fumihito Arai contributed equally to this work

1 Department of Micro-Nano Systems Engineering, Nagoya University, Furo-cho, Chikusa-ku, Nagoya 464-8603, Japan
}

Full list of author information is available at the end of the article despite human hand tremors measuring approximately $100 \mu \mathrm{m}[2,3]$. Furthermore, the retinal vessels lie on the curved surface and surgeons must find the target vessel based on their senses of vision and touch. Therefore, obtaining the skill required to perform microcannulation surgery takes a long time, and the consequent long evaluation and training of young doctors is one of the drawbacks of microcannulation. Another drawback of this approach is the evaluation of new medical equipment. Several medical equipment for this difficult surgery, such as surgical robots, have been proposed in recent years [4-6]. However, large barriers currently prevent commercialization of these new instruments, including the lengthy period required for clinical trials. Thus, a demand exists for methods to reduce the time to market for such equipment and shorten the training and evaluation periods required for surgeons.

At present, training and evaluation of this medical technique are done with animal samples such as swine eyes or chick embryos [4-10]. However, structural differences between individual animal samples are significant, so the 

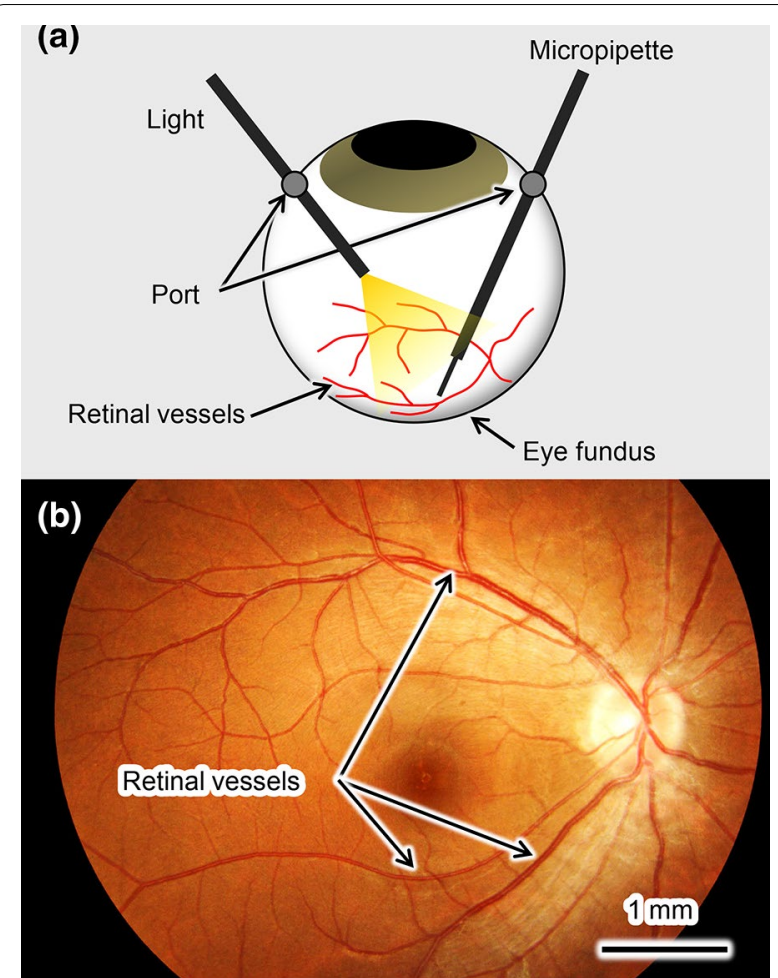

Fig. 1 Central retinal vein occlusion: a schematic of microcannulation and $\mathbf{b}$ optical-microscope photograph of retinal vessels

conditions of evaluation or training differ for each trial. Furthermore, using such animal samples in an actual operating room presents contamination risks. Therefore, a high demand exists for a reproducible method of evaluating vitreoretinal surgery techniques that can be used in an actual operating room.

One response to this demand is a surgical simulator. Basically, two types of surgical simulators exist: a computer-based virtual reality (VR) simulator [11-15], and a mock-up simulator made of artificial materials [16-20]. Once electronic data from the target parts are acquired, the VR simulator can construct the target parts with high reproducibility. However, mimicking the physical sensation of touch or the texture of a retinal vessel with the VR simulator is very difficult, and such sensations are critical for the surgeon performing vitreoretinal surgery. Additionally, the effect of new medical equipment on the human body is difficult to evaluate because interactions between the human body and medical equipment are too complex to completely reproduce with current computer technology. Thus, VR simulators are not suitable for evaluating medical techniques.

The second approach of a mock-up simulator has the advantage that it can be physically touched, allowing the user to learn the requisite sensations of touch or texture. Mock-up simulators use individual samples that are highly reproducible because of their well-controlled fabrication methods. Mimicking blood flow can also be implemented by circulating a liquid in the vessel models by connecting them to external tubes and pumps. Furthermore, selection of appropriate materials allows the simulator to be sterilized and facilitate the reproduction of the effects of such medical equipment on the human body. Thus, mock-up simulators can be used in actual operating rooms to evaluate the performance of medical equipment. Therefore, the goal of this research is to develop a mock-up surgical simulator to allow evaluation of medical techniques (e.g., surgical skills) or the performance of medical equipment.

Several studies have already been published discussing mock-up simulators for various sections of blood vessels [17-20]. For large vessels (in $\mathrm{mm}$ ) such as the coronary artery or arteries in the brain, such simulators can benefit from 3D printing technology. To simulate catheterization surgery, Ikeda et al. fabricated a millimeter-sized 3D vessel model made of polydimethylsiloxane (PDMS) using the lost-wax method $[17,18]$. However, fabricating a model of fine vessels of size below $100 \mu \mathrm{m}$ (e.g., retinal vessels) is difficult with this method. Photolithographybased fabrication techniques can be applied to fabricate models of smaller vessels. For example, Nakano et al. fabricated a microchannel that mimics a fine blood vessel with sizes down to about $10 \mu \mathrm{m}$ using photolithography techniques and PDMS molding [19]. Although photolithography offers sufficient resolution for the retinal vessel model, it can only be applied to $2 \mathrm{D}$ structures. The target of the present study, however, is to model retinal vessels of size about $100 \mu \mathrm{m}$ and that lie on the eye fundus, which forms a concave curved surface. Therefore, both the above-mentioned fabrication methods are difficult to apply in this case (i.e., a retinal vessel model with microchannels on a curved surface).

We thus propose a retinal vessel model with microchannels smaller than $100 \mu \mathrm{m}$ that lie on a concave curved surface. This retinal vessel model is fabricated by combining laser-lithography-based fabrication techniques, PDMS molding, and hydraulic transfer techniques. Furthermore, the microchannel wall thickness is controlled with $10 \mu \mathrm{m}$ accuracy to mimic the physical human sensation of touching retinal vessels. We circulate liquid through the fabricated microchannels and simulate a microcannulation procedure. We also measure the force applied to the model during the simulated surgery to demonstrate the usefulness of the proposed model not only for qualitative evaluation but also for quantitative evaluation of medical techniques. 


\section{Concept}

Figure 2 shows the concept of the proposed retinal vessel model, which consists of three layers. The bottom layer is a curved surface structure (curvature diameter of $24 \mathrm{~mm}$ ) that mimics the human eye. The middle layer is a PDMS sheet with microchannels less than $100 \mu \mathrm{m}$ in size, which mimics the structure of human retinal vessels. Finally, the top layer consists of a thin PDMS sheet, whose thickness is controlled to within about $10 \mu \mathrm{m}$ to mimic the sensation of touching human retinal vessels.

First, microchannels in the middle layer are fabricated on a PDMS sheet by laser lithography techniques and PDMS molding. Next, this middle layer is transferred to the concave bottom layer using a hydraulic transfer technique. The result is a fine vessel structure (vessels less than $100 \mu \mathrm{m}$ in diameter) superposed on the concave surface. Third, again using hydraulic transfer, the $10-\mu \mathrm{m}$-thick top layer is laid on the middle layer. Thus, the wall thickness of the top of the microchannels can be controlled to mimic the sensation of touching a retinal vessel. Furthermore, the mechanical properties of the model, such as Youngs modulus or tear strength, can be tuned by changing the mixing ratio of the base resin and the curative reagent for PDMS. Therefore, this model offers both the physical structure of human retinal vessels and the tactile sensation of touching them.

All parts of the model consist of PDMS or glass, which can withstand high temperatures and sterilization. As a result, the model can be used in actual operating rooms. Additionally, the microchannels can be connected to external tubes, as shown in Fig. 2a, allowing liquid to be circulated through the microchannels for mimicking blood flow. This enables us to circulate liquid in the microchannel for mimicking blood flow. Thus, the surgical procedure of injecting thrombolytic drugs into veins with a micropipette can be simulated with the proposed model. Furthermore, the force applied during the simulation can be measured using a force sensor placed underneath the model. The force information can be used to quantitatively evaluate medical techniques. We thus expect the proposed model to strongly contribute to evaluating surgical techniques or medical equipment and in the training of surgeons for retinal vessel surgery.

\section{Fabrication}

\section{Design of microchannel}

First, we designed microchannels for mimicking retinal vessels based on a shape of real retinal vessels. We simplified shape of real retinal vessels as shown in Fig. 3 and determined sized of vessels according to Murray's law [21, 22]. Murray's law states that the cube of radius of a parent vessel equals the sum of the cubes of the radii of the daughters, as shown in Fig. 3a and following equation.

$$
R_{0}^{3}=R_{1}^{3}+R_{2}^{3}
$$

We determined the width of first channel $\left(W_{1}\right)$ as 150.0 $\mu \mathrm{m}$ (radius: $R_{1}=75.0 \mu \mathrm{m}$ ), and calculated the width of second channel $\left(W_{2}\right)$ as $119.1 \mu \mathrm{m}$ (radius: $R_{2}=59.5$ $\mu \mathrm{m})$ according to Murray's law. Similarly, we calculated the width of third and fourth channels as $W_{3}=94.5$ $\mu \mathrm{m}$ (radius: $R_{3}=47.2 \mu \mathrm{m}$ ), and $W_{4}=75.0 \mu \mathrm{m}$ (radius: $\left.R_{4}=37.5 \mu \mathrm{m}\right)$, respectively.

\section{Mechanical characteristics of PDMS}

Second, we confirmed that the mechanical characteristics of PDMS may be suitably controlled by changing the mixing ratio of the main resin and curative reagent. In this study, we focus on Youngs modulus and tear strength

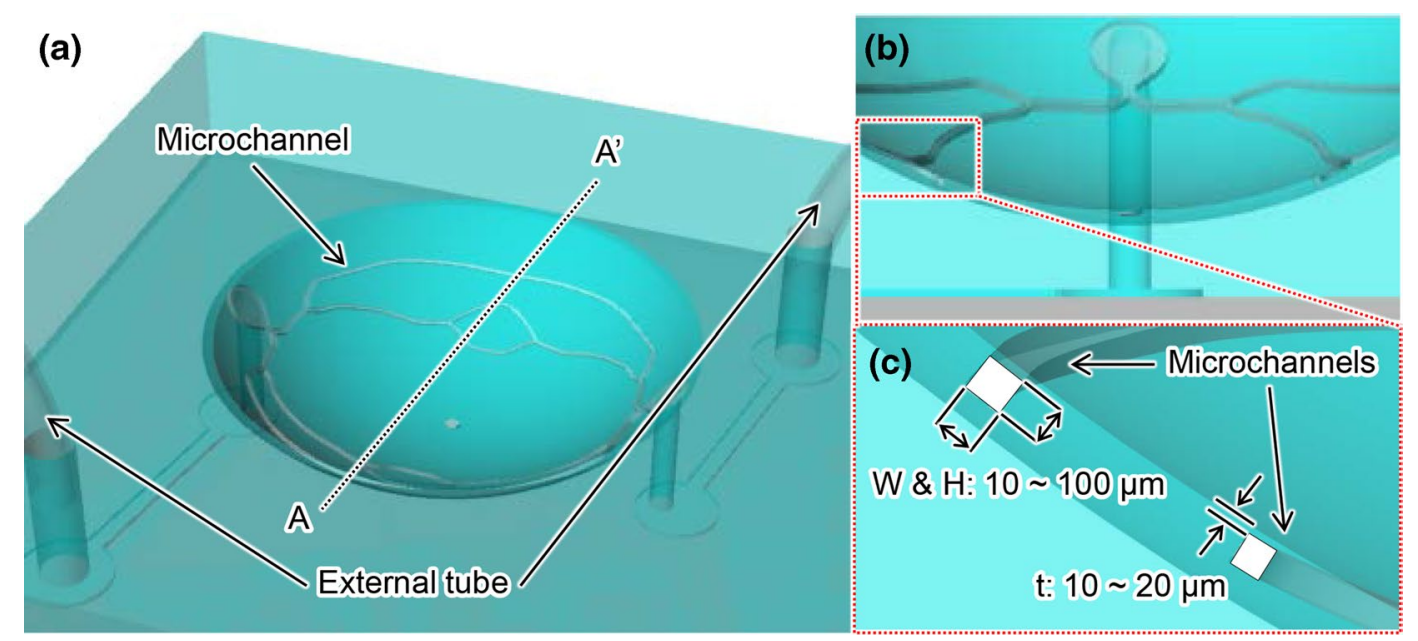

Fig. 2 Conceptual image of retinal vessel model on curved surface: a overview of model, $\mathbf{b}$ side view showing line A-A', and $\mathbf{c}$ close-up of side view 


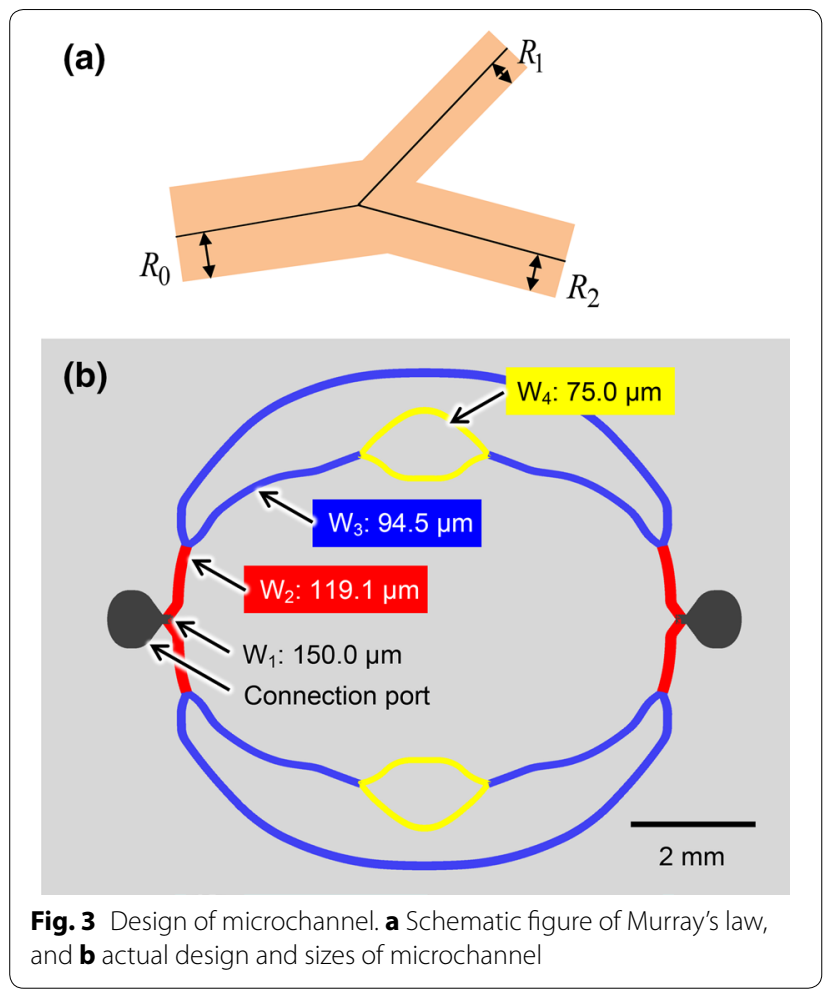

because our target is to simulate microcannulation, and these two parameters are thought to be strongly related to the sensation of touching retinal vessels and of feeling a puncture. Park et al. discussed the controllability of the mechanical characteristics of PDMS and used this approach to evaluate surgical skills for a coronary-artery bypass graft [23]. Although the coronary-artery target differs from the target of this study (i.e., retinal vessels), we use the same Youngs modulus and tear strength in our model as a reference (i.e., $0.13 \pm 0.02 \mathrm{MPa}$ and $0.6 \pm$ $0.13 \mathrm{~N} / \mathrm{mm}$, respectively).

The Youngs modulus and tear strength were evaluated based on the Japanese Industrial Standards (JIS) K6251 and K6252, which are equivalent to the International Organization for Standardization (ISO) 37 and 34. The dumb-bell test piece 7 and the angle test piece were used as samples. Each sample was pulled at $200 \mathrm{~mm} / \mathrm{min}$ and $500 \mathrm{~mm} / \mathrm{min}$. We fabricated samples with various mixing ratios (ratio of curative reagent to main resin) of 10 , 20, 33, 50 and $66 \mathrm{wt} \%$. The tensile tests were done three times for each mixing ratio.

Figure $4 \mathrm{a}$ and $\mathrm{b}$ show the measured Young's modulus and tear strength, respectively. We tuned the Youngs modulus from 0.14 to $1.14 \mathrm{MPa}$ and the tear strength from 0.68 to $1.70 \mathrm{~N} / \mathrm{mm}$. Considering the target values, we chose a mixing ratio of $66 \mathrm{wt} \%$ for this study. Furthermore, we performed preliminary sensory testing by
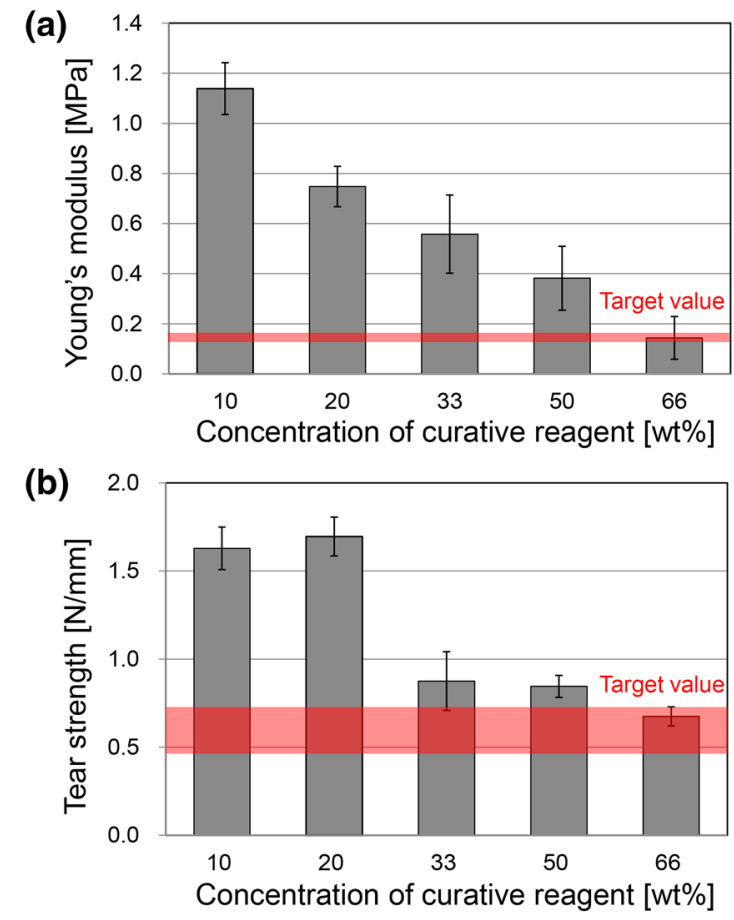

Fig. 4 Measured $\mathbf{a}$ Youngs modulus and $\mathbf{b}$ tear strength as a function of mixing ratio of the curative agent. The red areas indicate the target values

ophthalmologist about sensation of touch and puncture. By using flat vessel model, we confirmed that the PDMS with mixing ratio of $66 \mathrm{wt} \%$ are the most suitable for retinal vessel model.

\section{Hydraulic transfer of PDMS sheet}

To fabricate a retinal vessel structure on a curved surface, we hydraulically transferred the PDMS pattern. Hydraulic transfer is generally used for printing on curved surfaces $[24,25]$. By floating a printed film on water and pressing a curved surface onto the film, uniform printing on the curved surface is obtained. Retinal vessels on a curved surface are obtained by overlaying patterned PDMS sheets on a curved surface, as shown in Fig. 5. With this hydraulic transfer process, we can realize the fine microchannel with size of $\simeq 10 \mu \mathrm{m}$ on a curved surface, which is difficult to achieve with conventional fabrication techniques.

The details of fabrication are as follows:

1. Pattern SU-8 photoresist (Nippon Kayaku Co. Ltd, Tokyo, Japan) on a silicon surface by laser lithography. This pattern is used as a mold for the microchannels and the size of the microchannels can be locally controlled by adjusting the exposure conditions. 


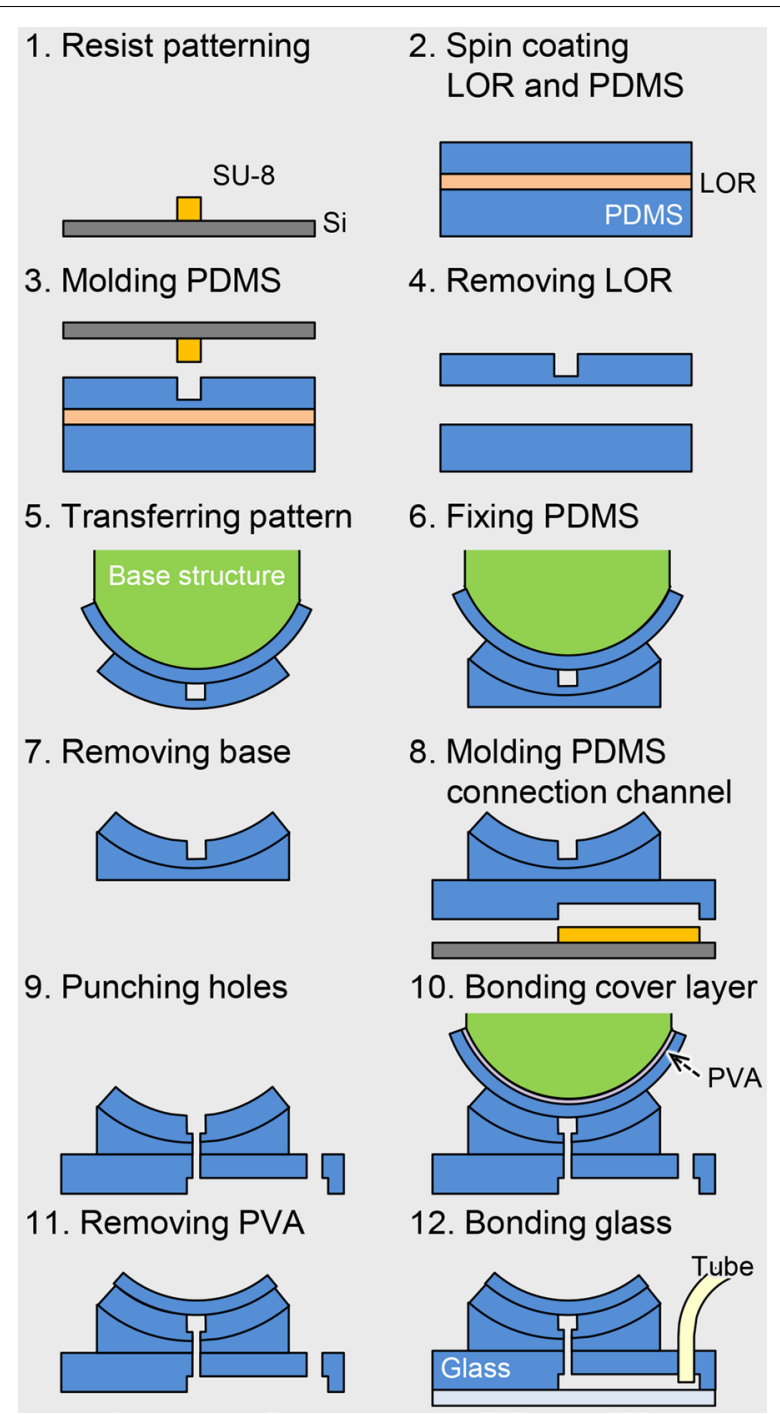

Fig. 5 Fabrication process for proposed retinal vessel model

2. Spincoat LOR (Nippon Kayaku Co. Ltd., Tokyo, Japan) and PDMS (Silpot 184, Dow Corning Toray Co. Ltd., Tokyo, Japan) onto the cured PDMS.

3. Push SU-8 mold ointo spin-coated PDMS and heat the ensemble to $85^{\circ} \mathrm{C}$ for $10 \mathrm{~min}$ with a hot plate.

4. Dissolve LOR with ethanol and remove PDMS sheet.

5. Transfer PDMS sheet to base structure by using hydraulic transfer. The base structure is made by a 3D printer (EDEN250, Stratasys Ltd.). The diameter of the base structure is $24 \mathrm{~mm}$, which is the average diameter of a human eye.

6. Transfer the PDMS sheet to the concave PDMS made by $3 \mathrm{D}$-printer mold. Prior to this step, treat both bonding surfaces with $\mathrm{O}_{2}$ plasma for surfaceactivated bonding of PDMS.

7. Remove base structure.

8. Pattern a connection channel at the bottom of the curved PDMS surface. The mold for the connection channel is fabricated by photolithography.

9. Punch holes into microchannel and connection channel to connect external tubes.

10. For a cover layer, bond the thin PDMS sheet to the curved surface. The polyvinyl alcohol (PVA) and thin PDMS sheet are coated onto the base structure by dip coating. The PVA is used to demold the thin PDMS sheet from the base structure.

11. Dissolve PVA with hot water and remove base structure.

12. Bond bottom glass to model to seal the connection channel and connect the external tubes.

In this fabrication process, the $10-\mu \mathrm{m}$-sized microchannel can be fabricated by using laser lithography (step 1 in Fig. 5). The patterning on a curved structure is done by using hydraulic transfer (steps 5 and 6). Furthermore, the thickness of the cover layer is controlled by changing the dip-coating conditions (step 10). This means that the wall thickness of the microchannels can be controlled to best mimic the sensation of touching retinal vessels. According to Ref. [26], the wall thickness of retinal vessels ranges in humans from 10 to $20 \mu \mathrm{m}$. We tentatively confirmed that this range can be covered by changing the drawing speed of the dip-coating process. In this study, we use a $20 \mu \mathrm{m}$ cover layer.

According to our proposed fabrication process, the cross section of the fabricated vessel model is square because the cross-section of the patterned photoresist is square. We have already fabricated the vessel model with circular vessel cross sections by using a reflow process with the patterned photoresist [19]. However, this approach requires additional processing steps, wihch increases the cost of fabrication. Therefore, this approach is not favored for commercial versions of this model. In addition, we made preliminary evaluations of both the square- and circular-cross-section microchannels. According to qualitative evaluations of these two models by medical doctors, no significant difference is apparent between the models with vessels of different cross-sectional shape. We therefore used vessels with square cross-sections in this study.

\section{Results}

The fabricated retinal vessel model is shown in Fig. 6. The channel was neither broken nor collapsed after the hydraulic transfer, as shown in Fig. 6b and c. We varied the width and height of the vessels from 75.0 to $119.1 \mu \mathrm{m}$ to mimic the size range of actual retinal vessels. Details of the design values and the measured results for the three typical parts of the samples are shown in Table 1 . The 


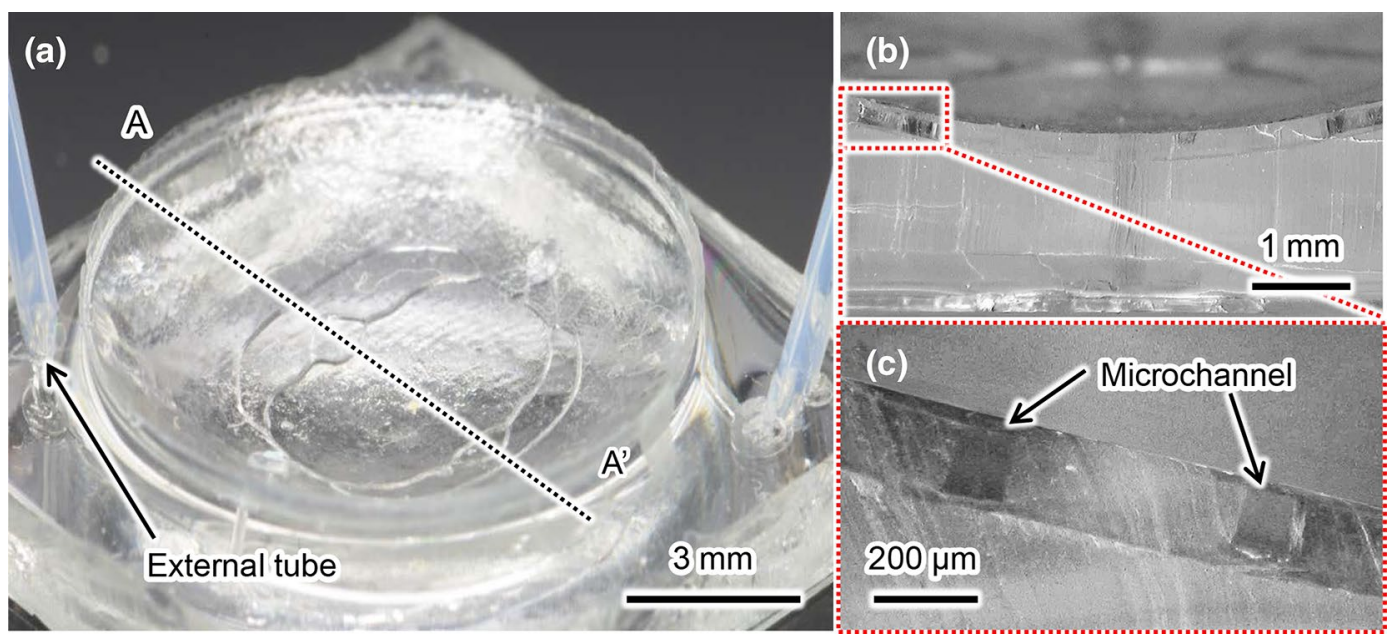

Fig. 6 Photographs of fabricated model: a overview, b cross-sectional view cut along line A-A', and c enlarged image of cross-sectional view

Table 1 Measurements of fabricated model $(\mathrm{N}=5)$

\begin{tabular}{lcccr}
\hline Design value & & & \multicolumn{2}{l}{ Fabricated results } \\
\cline { 5 - 5 } $\mathbf{W}(\boldsymbol{\mu m})$ & $\mathbf{H}(\boldsymbol{\mu m})$ & & $\mathbf{W}(\boldsymbol{\mu m})$ & $\mathbf{H}(\boldsymbol{\mu m})$ \\
\hline 119.1 & 119.3 & & $119.3 \pm 2.5$ & $124.9 \pm 4.2$ \\
94.5 & 94.9 & & $93.7 \pm 1.7$ & $99.8 \pm 3.4$ \\
75.0 & 74.8 & $73.2 \pm 1.1$ & $77.5 \pm 3.1$ \\
\hline
\end{tabular}

aspect ratio of these vessels is approximately 1.0. In addition, we evaluate the thickness of the cover-layer PDMS sheet, which is related to sensation of touching a retinal vessel. We measured the thickness at five points on a sample and the average value of these five thicknesses is $19.3 \pm 0.3 \mu \mathrm{m}$, which is close to the target value of $20 \mu \mathrm{m}$. The standard deviation of the thickness is less than $2 \%$ of the target value. Thus, we conclude that the proposed model can be fabricated with good reproducibility.

Next, we tested the circulation of liquid in the microchannel by injecting a blue liquid into the microchannel via the external tube connected to the model. The injected liquid circulated through the channel without leakage, as shown in Fig. 7 (Additional file 1).

Based on these results, we conclude that the proposed retinal vessel model was successfully fabricated on a concave surface. This fabrication method can thus be used to fabricate microchannels on concave surfaces.

\section{Discussion}

\section{Simulation of microcannulation}

Finally, we report a microcannulation procedure simulated by the fabricated retinal vessel model. The standard microcannulation procedure consists of the following four steps [27]:
1. Approach target retinal vessel with micropipette.

2. Puncture target vessel with micropipette.

3. Inject thrombolytic drug via the micropipette and hold micropipette in place for approximately $30 \mathrm{~s}$.

4. Withdraw micropipette from retinal vessel.

Additionally, we measured the vertical force applied to the model during this procedure by using a load cell placed underneath the model.

Photographs taken before and after puncturing (step 2) are shown in Fig. 8a and b, respectively. The micropipettes successfully puncture the microchannel and inject the liquid into the channel (Additional file 2). The vertical force applied during the procedure is shown in Fig. 8c and varies from approximately -150 to $180 \mathrm{mN}$. Here, negative values indicate that the force is applied downward towards the model (i.e., a pushing force). Similarly, positive values indicate a pulling force. Thus this model allows quantitative measurements of the force applied during the simulation of a microcannulation procedure.

\section{Future works}

We propose herein a retinal vessel model and describe how to fabricate the model. Further functionalization of the proposed model is also discussed.

Simulating entire steps of microcannulation surgery requires a whole-eye model. In this study, we focus on simulating the retinal vessels at the posterior segment of the eye. Our model can be used to simulate the puncture and injection processes in microcannulation surgery. However, other processes in an actual operation, such as insertion of forceps into the eyeball, should also be simulated. To do so would require a whole-eye model that can mimic the structures and mechanical characteristics of 

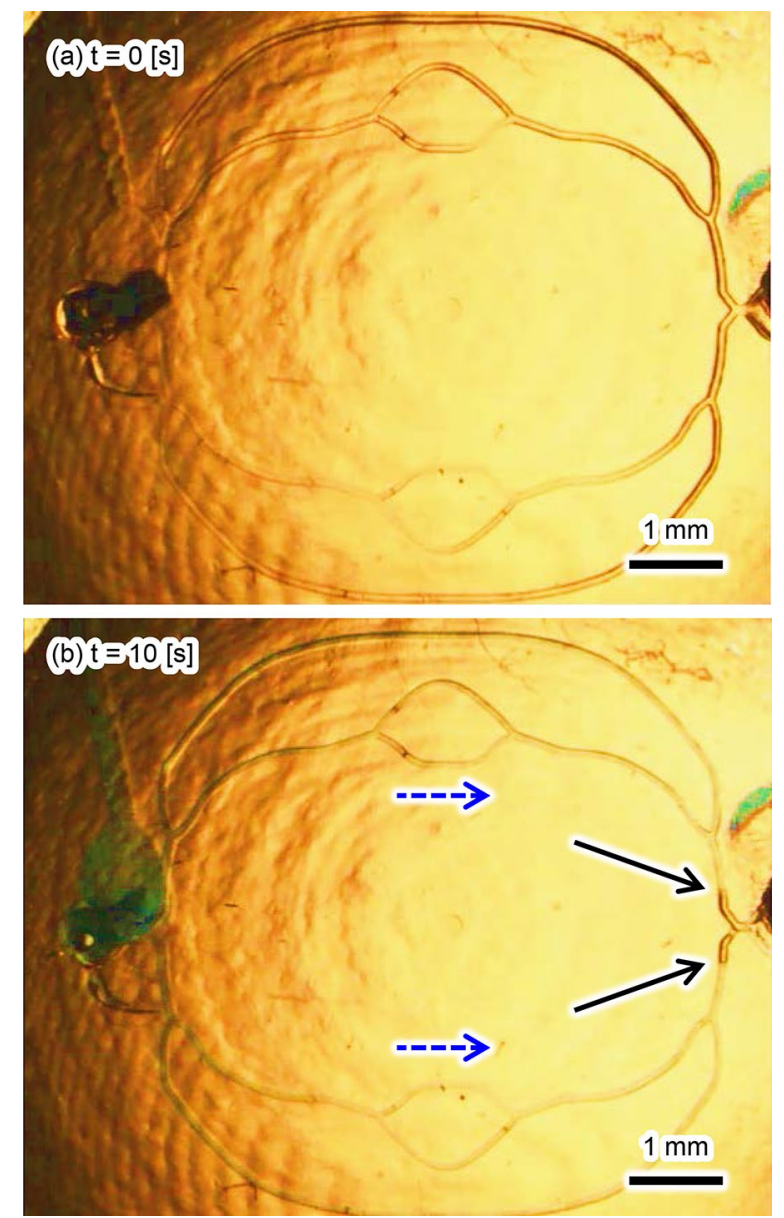

Fig. 7 Successive photographs of the circulation of liquid through the microchannel: a start of circulation and $\mathbf{b} 10 \mathrm{~s}$ later. The black arrows point to the air-liquid interface, whereas the blue dotted arrows show the direction of flow

whole parts of the human eyeball. The fabrication of such a complex 3D structure with the appropriate materials is planned in future work.

Furthermore, we demonstrate the measurement of applied force with the proposed model, as shown in Fig. 8c. Integrating such sensing functions into a mockup surgical simulator is of great use for quantitatively evaluating medical techniques. Other surgical procedures use thermal or electrical effects for treatments. Simulating the associated sensing functions (e.g., thermal or electrical sensors) is required to evaluate these surgical procedures. Such highly-functionalized surgical simulators will be reported in the near future.
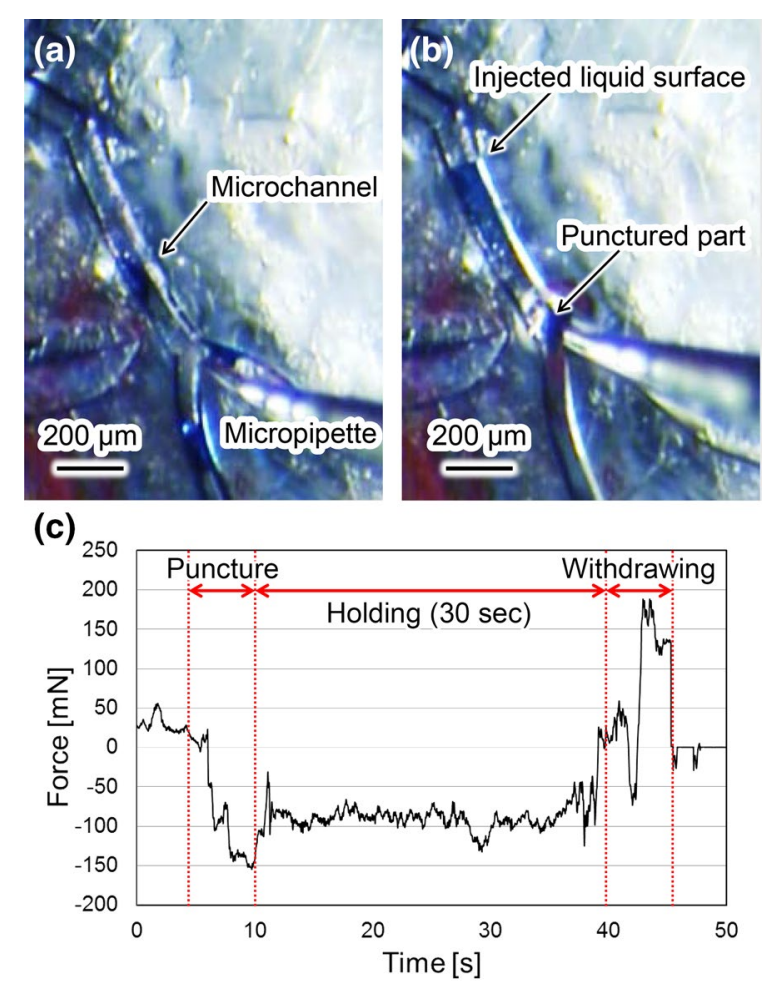

Fig. 8 Results of microcannulation simulation: photographs of retinal vessel model $\mathbf{a}$ before and $\mathbf{b}$ after puncture of retinal vessel by micropipette. These photographs were acquired by using an eye-surgery microscope. c Measured force applied during microcannulation procedure

\section{Conclusions}

We propose herein a retinal vessel model fabricated on a concave surface to simulate microcannulation. The structure of the proposed model is very reproducible and can be sterilized for use in actual operating rooms. The model is fabricated by using laser lithography, PDMS molding, and hydraulic transfer, and can create vessels as small as $10 \mu \mathrm{m}$ in size. Furthermore, we confirmed that liquid can be circulated through the fabricated microchannel. In addition, we simulate the puncture and injection processes of microcannulation. We also measure the applied force by using a force sensor placed underneath the model. Such sensing is quite important to quantitatively evaluate surgical skills or the performance of medical equipment. The proposed retinal vessel model and the associated sensing function strongly contribute to evaluating medical techniques, which are vital to ensure highquality and safe medical procedures. 


\section{Additional files}

Additional file 1: Circulation. Movie file of liquid circulation to fabricated retinal vessel model (Fig. 7)

Additional file 2: Puncture. Movie file of simulation of puncture and injection process with fabricatedretinal vessel model (Fig. 8).

\section{Authors' contributions}

All authors performed conception and design of the study. IK performed collection of data, analysis and interpretation of data, TH and FA performed drafting of the manuscript and all authors performed critical revision of the manuscript. All authors read and approved the final manuscript.

\section{Author details}

${ }^{1}$ Department of Micro-Nano Systems Engineering, Nagoya University, Furo-cho, Chikusa-ku, Nagoya 464-8603, Japan. ${ }^{2}$ Department of Mechanical Engineering, School of Engineering, The University of Tokyo, 7-3-1 Hongo, Bunkyo-ku, Tokyo 113-8656, Japan. ${ }^{3}$ Department of Ophthalmology, School of Medicine, The University of Tokyo, 7-3-1 Hongo, Bunkyo-ku, Tokyo 113-8655, Japan.

\section{Acknowledgements}

This study was supported by Grant-in-Aid of the program Impulsing Paradigm Change through Disruptive Technologies Program (ImPACT).

\section{Competing interests}

The authors declare that they have no competing interests.

Received: 29 April 2016 Accepted: 31 August 2016

Published online: 13 September 2016

\section{References}

1. Tang WM, Han DP (2000) A study of surgical approaches to retinal vascular occlusions. Arch Ophthalmol 118(1):138-143

2. Singh S, Riviere C (2002) Physiological tremor amplitude during retinal microsurgery. In: Bioengineering conference, 2002. Proceedings of the IEEE 28th Annual Northeast. IEEE, New York, p 171-172

3. Riviere CN, Rader RS, Thakor NV (1998) Adaptive cancelling of physiological tremor for improved precision in microsurgery. IEEE Trans Biomed Eng 45(7):839-846

4. Ida Y, Sugita N, Ueta T, Tamaki Y, Tanimoto K, Mitsuishi M (2012) Microsurgical robotic system for vitreoretinal surgery. Int J Comput Assist Radiol Surg 7(1):27-34

5. Becker BC, Voros S, Lobes L, Handa JT, Hager GD, Riviere CN (2010) Retinal vessel cannulation with an image-guided handheld robot. In: Engineering in medicine and biology society (EMBC), 2010 annual international conference of the IEEE. IEEE, New York, p 5420-5423

6. Ueta T, Yamaguchi Y, Shirakawa Y, Nakano T, Ideta R, Noda Y, Morita A, Mochizuki R, Sugita N, Mitsuishi M (2009) Robot-assisted vitreoretinal surgery: development of a prototype and feasibility studies in an animal model. Ophthalmology 116(8):1538-1543

7. Olsen TW, Feng X, Wabner K, Csaky K, Pambuccian S, Cameron JD (2011) Pharmacokinetics of pars plana intravitreal injections versus microcannula suprachoroidal injections of bevacizumab in a porcine model. Invest Ophthalmol Vis Sci 52(7):4749

8. Huang W, Arai F, Kawahara T (2015) Egg-in-cube: design and fabrication of a novel artificial eggshell with functionalized surface. Plos One 10(3):0118624
9. Leng T, Miller JM, Bilbao KV, Palanker DV, Huie P, Blumenkranz MS (2004) The chick chorioallantoic membrane as a model tissue for surgical retinal research and simulation. Retina 24(3):427-434

10. Cox M, Irby DM, Reznick RK, MacRae H (2006) Teaching surgical skillschanges in the wind. New Engl J Med 355(25):2664-2669

11. Stergiopulos N, Young D, Rogge T (1992) Computer simulation of arterial flow with applications to arterial and aortic stenoses. J Biomech 25(12):1477-1488

12. Fuchs J, Warmann SW, Szavay P, Kirschner HJ, Schäfer JF, Hennemuth A Scheel-Walter HG, Bourquain H, Peitgen HO (2005) Three-dimensional visualization and virtual simulation of resections in pediatric solid tumors. J Pediatr Surg 40(2):364-370

13. Taylor CA, Draney MT (2004) Experimental and computational methods in cardiovascular fluid mechanics. Annu Rev Fluid Mech 36:197-231

14. Seymour NE, Gallagher AG, Roman SA, OBrien MK, Bansal VK, Andersen DK, Satava RM (2002) Virtual reality training improves operating room performance: results of a randomized, double-blinded study. Ann Surg 236(4):458

15. Kneebone $R$ (2003) Simulation in surgical training: educational issues and practical implications. Med Educ 37(3):267-277

16. Derossis AM, Fried GM, Abrahamowicz M, Sigman HH, Barkun JS, Meakins JL (1998) Development of a model for training and evaluation of laparoscopic skills. Am J Surg 175(6):482-487

17. Ikeda S, Arai F, Fukuda T, Negoro M, Irie K (2005) An in vitro patient specific biological model of the cerebral artery reproduced with a membranous configuration for simulating endovascular intervention. J Robot Mechatron 17(3):327-333

18. Matsushima M, Tercero C, Ikeda S, Fukuda T, Arai F, Negoro M, Takahashi I (2011) Photoelastic stress analysis in blood vessel phantoms: threedimensional visualization and saccular aneurysm with bleb. Int J Med Robot Comput Assist Surg 7(1):33-41

19. Nakano T, Itoyama T, Yoshida K, Sawada Y, Ikeda S, Fukuda T, Matsuda T, Negoro M, Arai F (2010) Multiscale fabrication of a transparent circulation type blood vessel simulator. Biomicrofluidics 4(4):046505

20. Tanaka S, Harada K, Ida Y, Tomita K, Kato I, Arai F, Ueta T, Noda Y, Sugita N, Mitsuishi M (2014) Quantitative assessment of manual and robotic microcannulation for eye surgery using new eye model. Int J Med Robot Comput Assist Surg 11(2):210-217

21. Murray CD (1926) The physiological principle of minimum work applied to the angle of branching of arteries. J Gen Physiol 9(6):835-841

22. Sherman TF (1981) On connecting large vessels to small. The meaning of murray's law. J Gen Physiol 78(4):431-453

23. Park Y, Shinke M, Kanemitsu N, Yagi T, Azuma T, Shiraishi Y, Kormos R, Umezu M (2009) A surgical training simulator for quantitative assessment of the anastomotic technique of coronary artery bypass grafting. In: 13th International conference on biomedical engineering. Springer, Berlin, $\mathrm{p}$ 1179-1182

24. Zhang Y, Yin C, Zheng C, Zhou K (2015) Computational hydrographic printing. ACM Trans Graph 34(4):131

25. Masuda T, Yamagishi Y, Takei N, Owaki H, Matsusaki M, Akashi M, Arai F (2013) Three-dimensional assembly of multilayered tissues using water transfer printing. J Robot Mechatron 25(4):690-697

26. Muraoka Y, Tsujikawa A, Kumagai K, Akiba M, Ogino K, Murakami T, AkagiKurashige Y, Miyamoto K, Yoshimura N (2013) Age-and hypertensiondependent changes in retinal vessel diameter and wall thickness: an optical coherence tomography study. Am J Ophthalmol 156(4):706-714

27. Weiss JN, Bynoe LA (2001) Injection of tissue plasminogen activator into a branch retinal vein in eyes with central retinal vein occlusion. Ophthalmology 108(12):2249-2257 